\title{
Contribution à l'étude écologique de la malacofaune de la zone Sud de la région de Tlemcen (Algérie)
}

\section{DAMERDJI Amina}

Département de biologie, Faculté des sciences, Université Aboubekr BELKAID-Tlemcen, Algérie

*Correspondance, courriel : damerdii_halim@yahoo.fr

\section{Résumé}

Le milieu que nous nous proposons d'étudier, occupe la partie sud de la région de Tlemcen. Il se caractérise par un bioclimat semi-aride. Le but principal de notre travail est de faire un inventaire faunistique sur deux formations végétales différentes du point de vue cortège floristique. Cette étude est réalisée dans 2 communes : Sebdou et ElAricha. Deux stations y sont prospectées respectivement : Sidi-Moussa (garrigue) et ElAovedj (maquis).

La $1^{\text {ère }}$ station comporte les espèces végétales telles: Chamaerops humilis, Marrubium vulgare, Stipa tenacissima, Artemesia herba alba, Asparagus acutifolius et Thymus ciliatus. La seconde comprend l'espèce arborée (Pinus halepensis) et des espèces arbustives et herbacées telles Atractylis et Marrubium.

Les échantillonnages sont effectués d'avril 2004 à janvier 2005, répartis en 20 prélèvements. La richesse spécifique des Gastéropodes est estimée à 8 espèces. Une étude comparative concernant les Gastéropodes est réalisée dans les 2 stations. Les variations saisonnière et mensuelle sont données. L'étude statistique a été réalisée par les indices écologiques de composition et de structure.

Mots-clés : Malacofaune, diversité, écologie, indices écologiques, zone sud, région de Tlemcen (Algérie). 


\section{Abstract \\ Contribution to the ecological study of the malacofauna in southern part to the Tlemcen region (Algeria)}

The area that we propose to study, occupies the Southern part of Tlemcen. It is characterized by an arid semi bioclimatic.

The principal goal of our work to make faunistic inventory on a vegetable formation. This study is carried out in two communes (Sebdou and El-Aricha).

Two stations are prospected there respectively; Sidi Moussa (scrubland) and El-Aouedi (Maquis). The first station comprises the vegetable species dominated such: Chamaerops humilis, Marrubium vulgare, Stipa tenacissima, Artemisia herba alba, Asparagus acutifolius and Thymus ciliatus.

The second incluted the species raised (Pinus halepensis) and of the shrubby species and herbaceous such: Atractylis and Marrubium.

The echantillonnages has been affected in april 2004 with january 2005, divided into 20 taking away.The specific richness of Gastropoda is estimated at 8. A comparative faunistic study is carried out in the two stations. The importance's seasonal and monthly are given. The statistical study was carried out by the ecological indications.

Keywords : Malacofauna, diversity, ecology, ecological indications, southern part, region's Tlemcen, Algeria.

\section{Introduction}

Pour ce qui concerne les travaux sur la faunistique dans la région de Tlemcen plusieurs d'entre eux ont été entrepris sur différentes espèces de plantes-hôtes. En effet dans la région steppique située au Sud de Tlemcen une étude bioécologique sur la faune de Stipa tenacissima L. (Poacées) comprenant notamment des Gastéropodes [1] a précédé une publication sur les Arthropodes dans la même région [2]. Des études bioécologiques sur la faune sont entreprises par la suite sur le "diss" Ampelodesma mauritanicum (Poiret) Durd et Shinz, 1895 (Poacées) dans la région de Tlemcen [3,4], sur le thym Thymus ciliatus Desf. (Lamiacées) [5] et sur le doum Chamaerops humilis Linné toujours aux alentours de Tlemcen [6-8]. Sur le Genêt (Fabacées), un inventaire malacologique est réalisé par $A$. Damerdji et $A$. Diedid [9]. Sur la malacofaune du romarin Rosmarinus officinalis, une étude complète a été effectuée par $A$. Damerdji et al. [10].

Des auteurs ont travaillé sur la taxonomie et la biogéoraphie des Gastéropodes de la péninsule ibérique et des îles baléares [11-13].

D'autres auteurs ont effectué des études sur les Mollusques terrestres dans des régions 
bien particulières telles la vallée de Najerilla [14] la commune de Valence $[15,16]$.

B. Gomez [11] a dressé un catalogue des espèces de la région ibérique.

Bigot et Aguesse [17] traitent des variations de structure de 7 écosystèmes méditerranéens hautement caractéristiques du delta du Rhône (Camargue).

S. Bechlaghem [18] a essayé d'apporter sa contribution dans l'étude faunistique de deux stations de la zone steppique ce qui nous a ramené à réaliser une étude faunistique assez poussée dans cette zone méridionale de la région de Tlemcen. L'objectif essentiel de ce travail est une recherche sur la malacofaune de la zone méridionale de la région de Tlemcen, ce qui justifie le présent manuscrit.

\section{Matériel et méthodes}

\section{2-1. Stations}

Pour réaliser ce travail, 2 stations situées dans la zone Sud de Tlemcen $\left(1^{\circ} 00^{\prime} \mathrm{W}-1^{\circ} 30^{\prime} \mathrm{W}\right.$; $34^{\circ} 15^{\prime} \mathrm{N}-34^{\circ} 45^{\prime} \mathrm{N}$ ) sont prises en considération (Figure 1).

La première (celle de Sidi-Moussa) est localisée dans la zone de Sebdou et la seconde (celle d'El-Aovedi) dans la zone d'El-Aricha. Elles se différencient par la morphologie, la végétation et l'altitude.

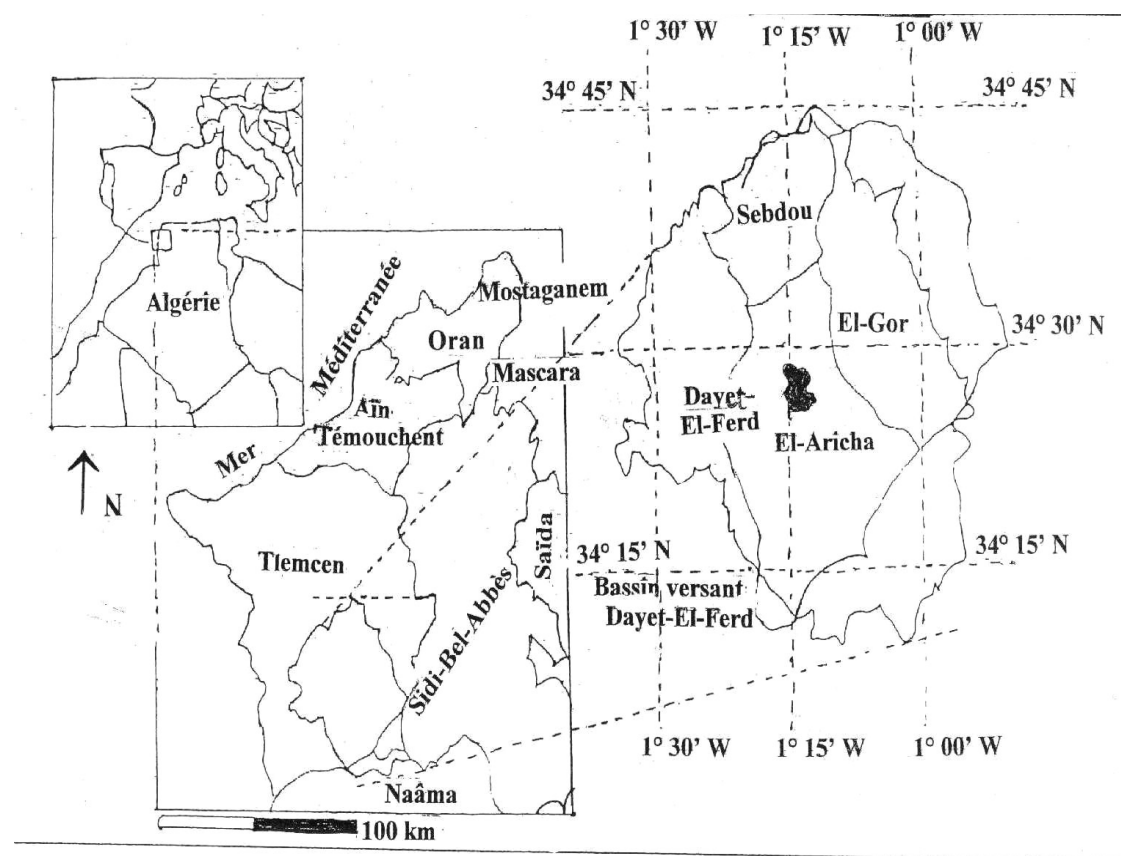

Figure 1 : Situation géographique de la zone sud de Tlemcen 


\section{2-1-1. Station $n^{\circ} 1:$ Sidi-Moussa (Figure2)}

Cette station d'étude se trouve entre la zone de Sidi- Aïssa à l'ouest et la R.N n ${ }^{\circ} 22$ au sud, elle est entourée par une zone forestière. Elle est à une altitude de $900 \mathrm{~m}$ et une pente supérieure à $15 \%$ et un taux de recouvrement de 20 à $30 \%$. Les espèces végétales qui dominent sont : Stipa tenacissima (Poacées), Artemisia herba alba (Composées), Chamaerops humilis (Palmacées), Marrubium vulgare (Labiées), Asparagus acutifolius (Liliacées) et Thymus ciliatus (Lamiacées).

Nous avons trouvé dans cette station d'autres espèces arbustives et herbacées qui ont une abondance-dominance et sociabilité de 1-1 qui sont : Echium vulgare (Borraginacées), Palena spinosa, Atractylis carduis (Composées), Sinapsis arvensis (Crucifères), Calendula arvensis (Composées), Reseda alba (Résédacées), Pelargonium asperum (Géraniacées), Bromus rubens (Graminées), Daucus carota (Apiacées), Malva sylvestris (Malvacées), Hordeum murinum (Graminées), Atractylis pycnocephalus (Composées), Anagalus arvensis (Primulacées), Asteriscus maritimus (Astéracées) et Plantago lagopus (Plantaginacées).

\section{2-1-2. Station $n^{\circ} 2$ : El-Aovedj (Figure 2)}

Elle est délimitée par la R.N. $n^{\circ} 22$ à l'ovest et à l'est par un champ de blé, et les limites nord par les agglomérations d'El-Aouedj et au sud par un secteur d'arbres de Pinus halepensis (Pinacées). Elle est caractérisée par une pente ne dépassant pas $5 \%$ et une altitude de $1250 \mathrm{~m}$ et un taux de recouvrement de 40 à $50 \%$. Les espèces végétales qui dominent sont : Pinus halepensis (Pinacées), Artemisia herba alba (Composées), Stipa torilis, Hordeum murinum (Graminées).

Cette station est peuplée par d'autres espèces herbacées et arbustives peu abondantes: Calendula arvensis (Composées), Reseda alba (Résédacées), Atractylis carduis (Composées), Echium vulgare (Borraginacées), Sinapsis arvensis (Crucifères), Malva sylvestris (Malvacées), Atractylis humilis (Composées), Lavandula multifida (Lamiacées) et Colchicum automnale (Liliacées).

Tableau 1: Données abiotiques et biotiques des 2 stations prospectées

\begin{tabular}{|l|c|c|l|c|}
\hline & Altitude (m) & Pente & $\begin{array}{l}\text { Etage } \\
\text { bioclimatique }\end{array}$ & $\begin{array}{c}\text { Recouvrement } \\
\text { Global }\end{array}$ \\
\hline $\begin{array}{l}\text { Station } \mathbf{N}^{\circ} \mathbf{1} \text { maquis } \\
\text { (Sidi-Moussa) }\end{array}$ & 900 & $15-25 \%$ & $\begin{array}{l}\text { Semi-aride à } \\
\text { hiver tempéré }\end{array}$ & $20-30 \%$ \\
\hline $\begin{array}{l}\text { Station } \mathbf{N}^{\circ} \mathbf{2} \text { garrigue } \\
\text { (El-Aouedi) }\end{array}$ & 1250 & $0-5 \%$ & $\begin{array}{l}\text { Semi-aride à } \\
\text { hiver frais }\end{array}$ & $40-50 \%$ \\
\hline
\end{tabular}




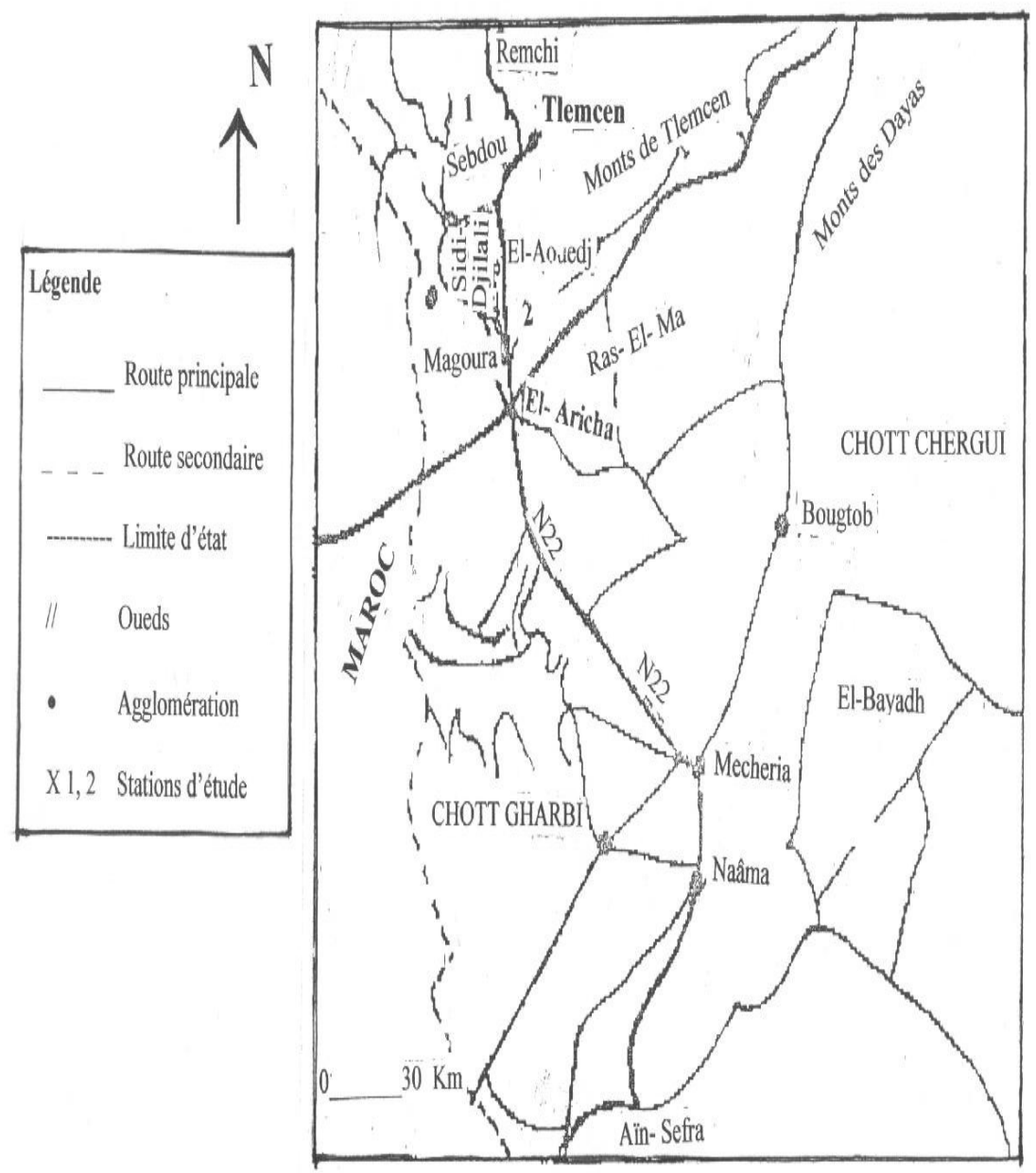

Figure 2 : Situation géographique des 2 stations d'étude

\section{2-2. Echantillonnage et identification}

Sur le terrain, 20 prélèvements sont effectués deux fois par mois depuis avril 2004 jusqu'en janvier 2005. Les échantillons sont ramenés au laboratoire où les individus vivants et les coquilles vides sont séparés. Ces dernières sont mises dans des sachets en matière plastique. Les espèces de petite taille sont conservées dans des tubes généralement en verre. Les caractères morphologiques et anatomiques n'ont pas la même importance du point de vue systématique. Cependant, il est tenu compte de la forme, de la taille, de la coloration et de l'ornementation de la coquille autant de différences morphologiques pouvant aider dans la détermination. Par ailleurs, les 
caractères anatomiques notamment, de l'appareil génital demeurent des critères déterminants pour l'identification des espèces. A ce propos la description morphologique s'appuie sur l'étude biosystématique des Mollusques Gastéropodes Pulmonés terrestres de la région de Tlemcen [19].

\section{2-3. Traitement des données}

Les données sont traitées en vue de la détermination de la fréquence d'occurrence, l'abondance ou fréquence centésimale et la densité.

La fréquence d'occurrence d'une espèce est le rapport exprimé en pourcentage, du nombre de prélèvements où cette espèce est notée au nombre total de prélèvements effectués [20] :

$$
\mathrm{F}=\frac{\mathrm{P}_{\mathrm{a}}}{\mathrm{P}} \times 100
$$

$F=$ fréquence d'occurrence de l'espèce. $P_{a}=$ nombre total de prélèvements contenant l'espèce prise en considération. $P$ est le nombre total de prélèvements faits. Selon Dajoz [20] on distingue : les espèces constantes $(F \geq 50 \%)$, les espèces accessoires $(25 \%<F$ $<50 \%$ ), les espèces accidentelles ( $F \leq 25 \%$ ).

L'abondance relative d'une espèce correspond au rapport du nombre des individus de cette même espèce au nombre total des individus toutes espèces confondues:

$$
A_{\text {rel }}=\frac{\mathrm{Na}}{\mathrm{Na}+\mathrm{Nb}+\mathrm{Nc}+\mathrm{N} \ldots} \times 100
$$

$A_{\text {rel }}=$ abondance relative de l'espèce prise en considération. Respectivement, $\mathrm{Na}, \mathrm{Nb}$, $\mathrm{Nc},=$ nombres des individus des espèces $a, b, c$.

L'abondance relative renseigne sur l'importance de chaque espèce par rapport à l'ensemble des espèces présentes.

La densité d'un peuplement est le nombre d'individus vivants de toutes les espèces par unité de surface.

$$
\mathbf{D}=\frac{N}{P}
$$

où $\mathrm{D}=$ Densité de l'espèce.

$N=$ Nombre total d'individus d'une espèce récoltée II a II dans le peuplement considéré.

$P=$ Nombre total des prélèvements effectués dans le peuplement considéré sur une surface de $100 \mathrm{~m}^{2}$.

Indice de diversité de Shannon-Weaver et équitabilité 
Le calcul de cet indice permet d'évaluer la diversité faunistique d'un milieu donné et de comparer entre elles, les faunes de différents milieux même lorsque les nombres d'individus récoltés sont très différents [20].

Les indices de Shannon-Weaver et d'équirépartition s'expriment par les formules suivantes: $H^{\prime}=-\sum q_{i} \log _{2} q_{i}$

$H_{\max }^{\prime}=\log _{2} S \quad$ (S= nombre d'espèces)

$H^{\prime}=$ Indice de diversité exprimé en bits

$\mathrm{H}_{\max }^{\prime}=$ Diversité maximale exprimé en bits

L'équitabilité (E) est définie comme le rapport de la diversité calculée à la diversité maximale.

$$
\mathrm{E}=\frac{\mathrm{H}^{\prime}}{\mathrm{H}_{\max }^{\prime}}
$$

\section{Résultats}

\section{3-1. Diversité des espèces malacologiques récoltées dans la zone Sud de la région de Tlemcen}

En nous basant sur la classification de [21,22] une liste systématique des espèces retrouvées est établie.

Liste des espèces de Gastéropodes Pulmonés recensées dans la zone sud de la région de Tlemcen dans deux stations d'avril 2004 en janvier 2005

Sphincterochilidae

Sphincterochila (Leucochroa) candidissima Draparnaud, 1801

Helicinae

Archelix juilleti Terver, 1839

Archelix wagneri Terver, 1839

Archelix zapharina Terver, 1839

Archelix lactea Michaud, 1831

Helicellinae

Helicella (Trochoïdea) pyramidata Draparnaud, 1801

Helicella (Xerophila) lemoinei (Debeaux) Kobelt, 1822

Subulinidae

Rumina decollata Linné, 1758

Les Gastéropodes comptent seulement 8 espèces réparties en trois familles respectivement celle des Sphincterochilidae, celle des Helicidae et des Subulinidae. 


\section{3-2. Variations saisonnières de la richesse spécifique des Gastéropodes}

Au printemps, les Gastéropodes présentent une richesse spécifique égale à 4 dans les deux stations. En été, les Gastéropodes sont toujours présents dans les 2 stations étudiées avec 3 espèces dans la station de Sidi-Moussa et 5 espèces dans la station d'ElAouedj. En automne, la richesse spécifique malacologique est de 5 dans chacune des stations (Figure 3). En saison hivernale, les Gastéropodes sont représentés par trois espèces dans chacune des deux stations.

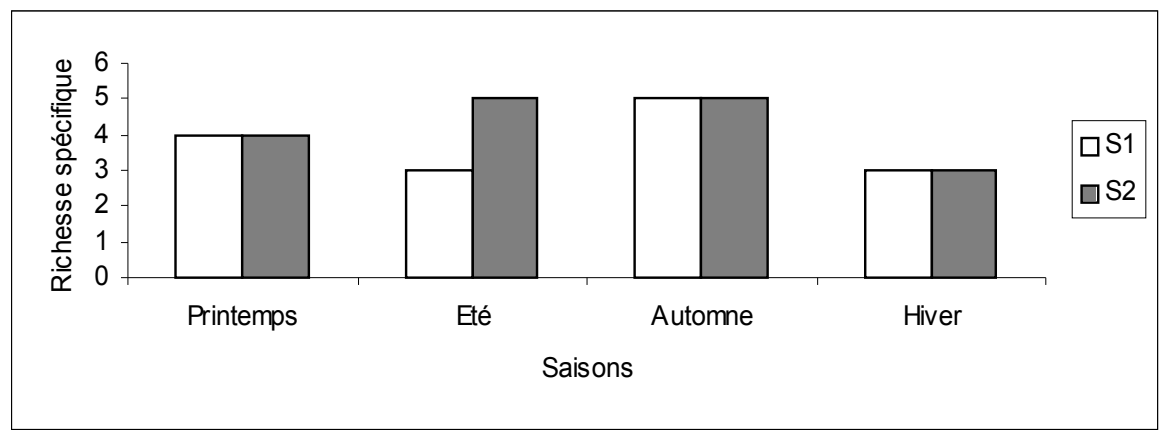

Figure 3 : Importance saisonnière selon la richesse spécifique des Gastéropodes dans les 2 stations

\section{3-3. Variations mensuelles de la richesse spécifique des Gastéropodes}

Au mois d'Avril, nous notons la présence des espèces malacologiques dans les 2 stations avec 2 espèces seulement (Figure 4). En Mai, les Gastéropodes augmentent dans la $2^{\text {ème }}$ station par rapport au mois précédent. En juin, la richesse spécifique est égale à 2 dans la station de Sidi-Moussa et à 4 dans la station d'El-Aouedj.

En juillet, nous notons l'augmentation des espèces malacologiques dans les 2 stations, 3 espèces sont retrouvées dans la $1^{\text {èr }}$ station et 5 espèces dans la seconde. En août, la richesse spécifique des Gastéropodes est en égalité dans les 2 stations avec une valeur de 3. En septembre, vu la diminution de température et l'augmentation d'humidité, 4 espèces sont rencontrées dans la ${ }^{\text {ère }}$ station et 5 espèces dans la seconde. En octobre, nous constatons que le nombre d'espèces malacologiques a diminué dans les 2 stations par rapport au mois précédent, 3 espèces pour la station de Sidi-Moussa et 2 espèces dans la station d'El-Aouedj. En novembre, les Mollusques sont présents dans les 2 stations avec 3 espèces dans chacune. En décembre, 5 espèces sont retrouvées dans la station de Sidi-Moussa et 4 espèces dans la station d'El-Aouedj. En dernier, le mois de 
janvier, les Gastéropodes sont moins importants dans les 2 stations par rapport au mois précédent.

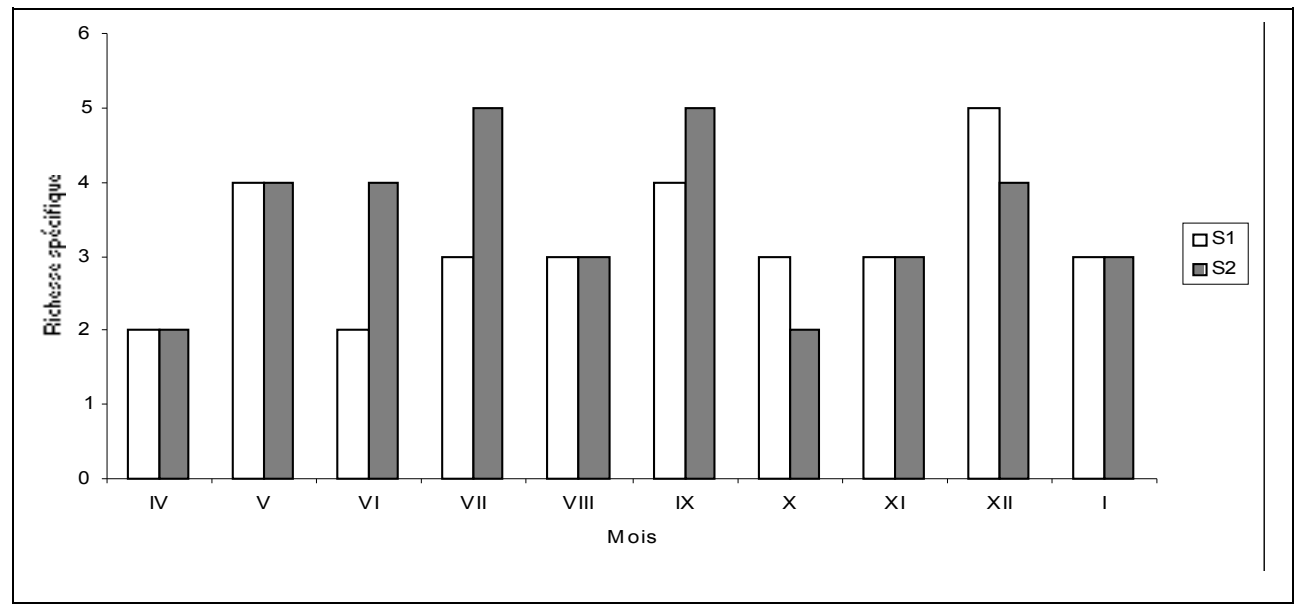

Figure 4 : Importance mensuelle des Gastéropodes récoltés dans les 2 stations (en effectifs)

\section{3-4. Variations mensuelles des nombres d'individus d'escargots recensés dans 2 stations de la zone sud}

Nous remarquons que les Gastéropodes sont présents pendant tous les mois, même les plus chauds. Le nombre d'individus dans la $1^{\text {ère }}$ station passe de 10 en avril à 86 en janvier (Figure 5). Dans la ${ }^{\text {2̀me }}$ station, l'effectif en août atteint 64 alors qu'en décembre il est de 90.

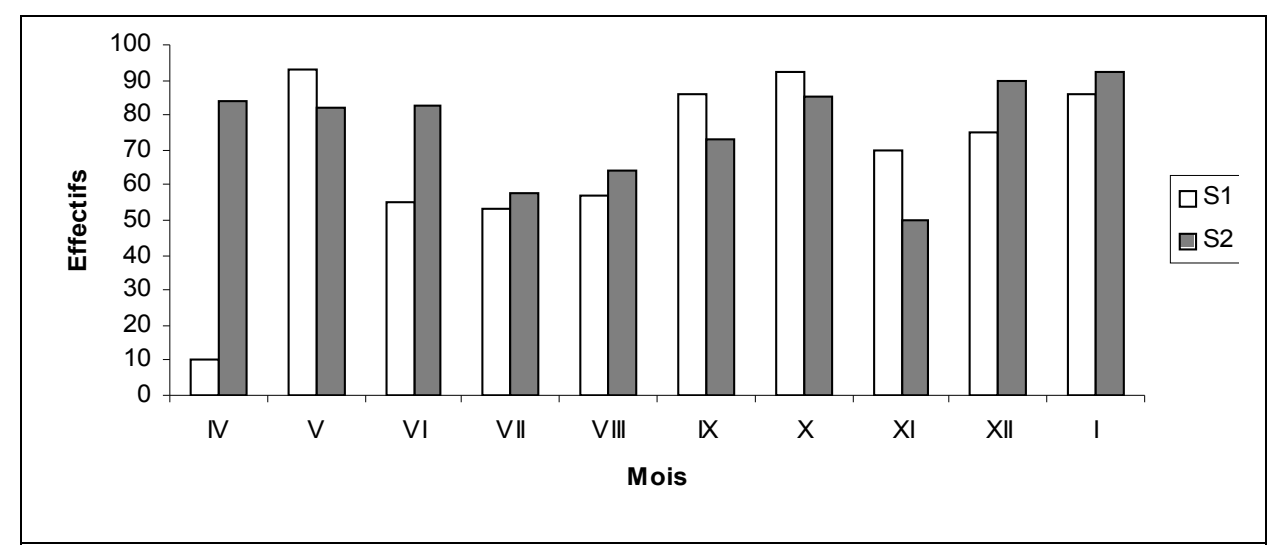

Figure 5 : Importance mensuelle des Gastéropodes récoltés dans les 2 stations (en effectifs) 


\section{3-5. Exploitation des résultats par des indices écologiques}

L'ensemble des espèces malacologiques inventoriées sont prises en considération pour le calcul des indices écologiques.

Tableau 2 : Fréquence d'occurrence, abondance, densité des espèces malacologiques

\begin{tabular}{|l||c|c|c|c|c|l|l|l||}
\hline \hline \multirow{2}{*}{$\begin{array}{l}\text { Espèces malacolo- } \\
\text { Giques considérées }\end{array}$} & \multicolumn{3}{|c|}{ Station 1 } & \multicolumn{3}{c|}{ Station 2} & Moyenne & \multirow{2}{*}{$\begin{array}{c}\text { Types } \\
\text { d'espèces }\end{array}$} \\
\cline { 2 - 10 } & F\% & A & D & F\% & A \% & D & (F\%) & \\
\hline Sphincterochila & 95 & 49.16 & 28 & 90 & 59.36 & 31.70 & 92.50 & Constante \\
\hline Archelix juilleti & 65 & 7.11 & 4.05 & 75 & 5.99 & 3.20 & 70 & Constante \\
\hline Archelix wagneri & 50 & 2.10 & 1.20 & 55 & 4.49 & 2.40 & 52.50 & Constante \\
\hline Archelix zapharina & 25 & 1.05 & 0.6 & 30 & 0.74 & 0.4 & 27.50 & Accessoire \\
\hline Archelix lactea & 5 & 0.08 & 0.05 & 5 & 0.18 & 0.10 & 5 & Très accidentelle \\
\hline Helicella pyramidata & 0 & 0 & 0 & 5 & 0.09 & 0.05 & 2.50 & Très accidentelle \\
\hline Helicella goundafiana & 0 & 0 & 0 & 5 & 0.09 & 0.05 & 2.50 & Très accidentelle \\
\hline Rumina decollata & 5 & 0.08 & 0.05 & 5 & 0.18 & 0.10 & 5 & Très accidentelle \\
\hline \hline
\end{tabular}

F \% : fréquences d'occurrence, $A \%$ : abondances relatives, $D$ : densité (sur $\left.100 \mathrm{~m}^{2}\right)$.

\section{3-5-1. Fréquence d'occurrence des espèces d'escargots}

Nous notons que 3 espèces sont constantes ayant une fréquence d'occurrence supérieure à $50 \%$. Ce sont : Sphincterochila candidissima, Archelix juilleti et $A$. wagneri. Comme espèce qualifiée d'accessoire nous avons retrouvé : Archelix zapharina. Pour ce qui est des espèces très accidentelles, nous comptons 4 espèces. II s'agit en fait de : Archelix lactea, Helicella pyramidata, H. goundafiana (Helicidae) et Rumina decollata (Subulinidae).

\section{3-5-2. Abondance relative et densité des escargots}

Nous avons discuté ces 2 indices ensemble. Ils éclairent sur la biologie et l'écologie de la malacofaune récoltée.

Sphincterochila candidissima (Sphincterochilidae) a une abondance de $49,16 \%$ et une densité de 28 sur $100 \mathrm{~m}^{2}$ dans la station de Sidi-Moussa, dans la station d'El-Aouedj son abondance est de $59,36 \%$ et une densité de 32 sur une surface de $100 \mathrm{~m}^{2}$. 


\section{3-5-3. Indice de diversité ou de Shannon-Weaver}

Cet indice de diversité est faible puisqu'il est égal à 0.26 dans chacune des deux stations. Deux espèces pullulent : il s'agit de Sphincterochila candidissima où l'effectif passe de 560 à 634 individus et Archelix juilleti atteint 81 individus dans la première station.

\section{3-5-4. Indice d'équirépartition appliqué aux espèces d'escargots}

L'équitabilité tend vers 0 , elle est inférieure à 0.5 dans les 2 stations étudiées. Dans la $\mathrm{l}^{\text {ère }}$ station, les effectifs des espèces sont différents, par exemple, Sphincterochila candidissima avec 560 individus et Archelix zapharina (Helicidae) avec seulement 12 individus. La seconde station, l'espèce Sphincterochila candidissima compte 634 individus et Archelix lactea seulement 2 individus.

\section{Discussion}

Sur le romarin [10], la richesse en Gastéropodes est de 18 espèces réparties entre 3 familles, celles des Sphincterochilidae, des Helicidae et des Subulinidae.

Sur Ampelodesma mauritanicum, M. Adjlani [3] a reconnu 13 espèces malacologiques alors que B. Bouhellou [6] en observe 18 sur Chamaerops humilis et que N. Kassemi [5] rencontre 19 espèces sur Thymus ciliatus. Sur le Calycotome spinosa (Genêt) nous dénombrons 21 espèces de Gastéropodes [9]. M. A. Khelil[1] avait noté une seule avec Leucochroa candidissima. Cette espèce actuellement dénommée Sphincterochila candidissima affectionne particulièrement les roches calcaires [19]. Cette dernière montre une forte adaptation morphologique pour son test épais et blanc qui doit la protéger des hautes températures pouvant sévir dans ces pelouses; elle se ferme en été par un épiphragme corné à l'abri duquel elle entre en diapause.

La bioécologie de la malacofaune retrouvée dans 2 stations (Hafir et Zarifelt) des monts de Tlemcen indique la présence de 34 espèces à Hafir et 19 espèces à Zarifelt pendant les années 1999 et 2000. Certains caractères conchyliologiques particuliers dont la taille et la couleur distinguent les espèces susceptibles de s'élever en altitude [23]. Pendant cette même période, une étude portant sur la répartition des espèces malacologiques du littoral (Ghazaouet), en passant par Tlemcen (centre urbain), les monts (Hafir et Zarifelt), Maghnia (plaine) jusqu'à la zone la plus méridionale et steppique (El-Aricha) a permis de relever 5 espèces communes dans ces différents écosystèmes : Sphincterochila candidissima (Sphincterochilidae), Archelix lactea, A. punctata et A. zapharina (Helicidae) ; et Rumina decollata (Subulinidae). II faut noter que 20 espèces sont considérées comme 
spécifiques comprenant 14 Helicidae [24]. A titre d'exemple, le $5^{\text {ème }}$ écosystème considéré (la zone steppique), nous y retrouvons 2 espèces d'Helicidae (Archelix bailloni et Helicella lemoinel).

Dans son essai d'écologie quantitative sur les Invertébrés de la Sansouire camarguaise, L. Bigot [25] indique en nombre d'espèces les mollusques recueillis dans les principaux milieux de Camargue. Dans la Sansouire, la richesse spécifique est estimée à 8. Les facteurs abiotiques et biotiques restent importants dans la variation de la taille des coquilles de Sphincterochila candidissima [26]. Cette espèce possède en milieu xérophile comme à El-Aricha une forte abondance. L.candidissima est très localisé en Camargue où il est connu du domaine de la tour du Valat et de ses environs [27]. La population de $L$. candidissima de la Camargue tend à montrer un net décollement des spires de sa coquille [28]. H. Enge/[29] signale sa présence principalement dans la Sansouire basse et salée à Arthrocnemum glaucum.

Par contre, E. pisana est commun dans toute la Camargue où ses tests s'amassent sous les Salicornia fruticosa et hébergent de nombreux invertébrés [27]. Selon L. Bigot [30] une faune importante représentant la majeure partie des ordres d'Invertébrés et à peu près tous les ordres d'insectes connus en Camargue, se réfugiait dans les coquilles vides. Ces tests jouent en effet un grand rôle en tant qu'abris. La faune y trouve un refuge idéal contre les basses températures de l'hiver et contre la canicule. Ces tests sont aussi utilisés comme source de nourriture, voire même de lieu de ponte et de métamorphose. C. F. Sacchi [31] a étudié l'écologie comparée des Gastéropodes pulmonés des dunes méditerranéennes et atlantiques.

Dans les stations à Romarin, les Gastéropodes ont une richesse égale à 12 espèces dans la quatrième station. Ainsi dans cette quatrième station, le nombre d'espèces a doublé en passant du printemps vers l'automne [10]. En saison hivernale, les Gastéropodes sont représentés par 3 espèces dans chacune des 2 stations. Ils préfèrent une certaine humidité ce qui permet d'expliquer leur présence dans les 4 stations [10].

B. Bouhellou [6] constate une diminution du nombre des espèces de Gastéropodes en juin. En septembre, les Gastéropodes associés au Romarin sont faiblement représentés en espèces dans la quatrième station [10]. La recrudescence d'activité s'explique par la diminution de la température moyenne et par l'augmentation de l'humidité à la suite des premières chutes de pluies automnales. En effet, 10 espèces sont à mentionner dans la quatrième station [10]. Cependant, en janvier, B. Bouhellou [6] a recensé sur Chamaerops humilis 6 espèces malacologiques dans la $l^{\text {ère }}$ station.

Dans le cas du Romarin, c'est la quatrième station qui est la plus importante, mais les fluctuations se font entre 125 individus en avril et 31 en décembre [10]. Dans l'étude effectuée sur Chamaerops humilis, B. Bouhellou [6] montre que les Gastéropodes occupent la seconde position du point de vue effectif avec 112 individus. La même 
constatation est faite par M. Adjlani [3] sur Ampelodesma mauritanicum avec 98 individus.

Le phénomène de " grappes $\|$ est une marque adaptative poussée vis-à-vis du milieu pour des espèces peu résistantes à l'état isolé. II se manifeste chaque année dans le delta du Rhône sur 2 ou 3 semaines au minimum et parfois sur plusieurs mois [17].

En considérant, la malacofaune associée au Romarin, cinq espèces sont constantes, ce sont Helicella pyramidata, H. virgata, Euparypha pisana, Eobania vermiculata et Rumina decollata 10].

Parallèlement sur Chamaerops humilis sur les 6 espèces prises en considération, 5 d'entre elles sont constantes. Elles sont très fréquentes au niveau de la $4{ }^{\text {ème }}$ station où l'humidité est suffisante comparativement aux autres stations [7]. A. Damerdii et $B$. Bouhellou [8], sur 9 espèces d'escargots notées sur Ampelodesma mauritanicum remarquent une seule espèce constante avec Macularia jourdaniana contre 6 espèces accessoires et 2 accidentelles dont Archelix juilleti et $A$. polita punctatiana.

L'abondance pour chacune des espèces Helicella pyramidata et $H$. goundafiana est de $0,09 \%$ et la densité égale à 0,05 . Ces 2 espèces sont absentes dans la station $n^{\circ} 1$. La même valeur de l'abondance dans les 2 stations pour les 2 espèces Archelix lactea (Helicidae) et Rumina decollata (Subulinidae) est égale à $0,08 \%$ dans la station $\mathrm{n}^{\circ} 1$ et l'abondance dans la station $n^{\circ} 2$ est de $0,18 \%$.

Concernant l'équirépartition relevée sur le Romarin c'est au niveau de la $3^{3 \text { me }}$ station que sa valeur est supérieure à 0,9 . Par conséquent, les effectifs des différentes espèces présentes ont tendance à être en équilibre entre eux (Macularia hieroglyphicula et Helicella pyramidata). II faut noter que même dans les stations 1 et 4, l'équitabilité est élevée puisqu'elle est presque égale à 0,8 [10].

\section{Conclusion}

L'étude bioécologique de la faune malacologique retrouvée dans la zone sud de Tlemcen a permis d'inventorier 8 espèces lors des 20 prélèvements effectués d'avril 2004 à janvier 2005. Quelque soit la saison, les Gastéropodes sont toujours présents dans les 2 stations étudiées. Les Gastéropodes sont retrouvés dans les deux stations même pendant les mois les plus chauds. Sur les 8 espèces malacologiques analysées, 3 espèces sont constantes, 1 est accessoire et 4 autres sont accidentelles. L'indice de Shannon Weaver présente la même valeur dans les 2 stations. II est de 0,26 bits. L'équirépartition indique un déséquilibre des peuplements. Enfin, si un certain nombre de résultats ont été dégagé au cours de cette étude, beaucoup de points restent à éclaircir, notamment la relation entre la strate végétale et la malacofaune qui y est recensée. 


\section{Références}

[1] - M. A. Khelil, ॥ Bioécologie de la faune alfatière dans la région steppique de Tlemcen II. Thèse Magister, Institut National Agronomique, El-Harrach, Alger, $n^{\circ} 9$ de la thèse (1984) 68p.

[2] - M. A. Khelil, ॥ Contribution à l'inventaire des Arthropodes de la biocénose de l'Alfa (Stipa tenacissima L., Graminées) dans la région de Tlemcen (Algérie) II. La défense des végétaux 257 (1989) 19-24.

[3] - M. Adjlani, II Contribution à l'étude bioécologique de la faune d'Ampelodesma mauritanicum (Poiret) Durd et Shinz, 1895 (Graminées) dans la région de Tlemcenll. Thèse Ingénieur Ecologie, Institut Sciences de la Nature, Université Aboubekr Belkaid, Tlemcen, (1998) 117p.

[4] - A. Damerdii et M. Adjlani, " Contribution à l'étude biocénotique d'une plante xérophyle : le Diss, en milieu semi-aride (région de Tlemcen) II. Communication Orale, Séminaire Adaptation des Organismes aux milieux steppique et saharien, 24-25 novembre 1999, Institut des Sciences de la Nature, Université Sciences et Technologie Houari Boumediène, Alger, (1999) 16p.

[5] - N. Kassemi, II Contribution à l'étude bioécologique de la faune de Thymus ciliatus Desf. (Thym) (Labiées) dans la région de Tlemcen II. Thèse Ingéniorat Ecologie et Environnement, Institut Sciences de la Nature, Université Aboubekr Belkaid, Tlemcen, (2001) $120 \mathrm{p}$.

[6] - B. Bouhellou, II Contribution à l'étude bio-écologique de la faune de Chamaerops humilis (Doum) (Monocotylédones, Palmacées) dans la région de Tlemcen II. Thèse Ingénieur Ecologie, Institut Sciences de la Nature, Université Aboubekr Belkaid, Tlemcen (1998) 93 pp.

[7] - A. Damerdji et B. Bouhellou, Faune des Invertébrés du Doum (Chamaerops humilis L.) : Inventaire-Indices écologiques dans la région de Tlemcen (Algérie). Communication orale, deuxième colloque international des chaires U.N.E.S.C.O.Gas Natural sur le développement durable du Maghreb : Diversités biologiques, écologiques, culturelles et environnementales, 28-30 avril 2002, Laghouat. (2002 $)$.

[8] - A. Damerdji et B. Bouhellou, Faune associée au Doum (Chamaerops humilis L.) dans la région de Tlemcen (Algérie) : Approche biocénotique. Poster, $\mathrm{I}^{\mathrm{èr}}$ Séminaire International Biologie et Environnement, 20-22 octobre 2002, Constantine. (2002b).

[9] - A. Damerdji et A. Djedid, La faune du Genêt (Calycotome spinosa Lamk.) dans la région de Tlemcen : Inventaire-Distribution spatio-temporelle des principaux groupes. Poster. lères Journées sur la Protection de l'Environnement. Université Aboubekr Belkaid-Tlemcen (2003) 28 et 29 Mai 2003. 
[10] - A. Damerdji, L. Ladjmi et S. Doumandji, "I Malacofaune associée à Rosmarinus officinalis L. (Labiatae): Inventaire et aperçu bioécologique près de Mansourah (Tlemcen, Algérie) II. Revue sciences et technologie, Constantine, Algérie.C- ${ }^{\circ} 23$, juin (2005) 11-20.

[11] - B. Gomez, Estudio sistematico y biogeografico de los Moluscos terrestres del Suborden Orthurethra (Gastropoda: Pulmonata: Stylommatophora) del Pais Vasco y regiones adyacentes, y catalago de las especias ibericas. Tesis Doctoral. Universitat Pais Vasco. (1988) 424 pp.

[12] - K. Altonaga, B. Gomez, R. Martin, C. E Prieto, A. I. Puente et A. Rallo, II Estudio fainistico y biogeografico de los Moluscos terrestres del norte de la Peninsula Iberica II. Parlamento Vasco, Vitoria, (1994) 503 p.

[13] - A. I. Puente., Estudio taxonomico y biogeografico de la Superfamilia Helicoidea Rafinesque, 1815 (Gastropoda : Pulmonata : Stylommatophora) de la Peninsula Iberica e Islas Baleares (1997), Tesis Doctoral. (U.P.V. / E.M.U.) 970 pp+33 lam.

[14] - A. Ortiz de Zarate, ॥ Descripcion de los Moluscos terrestres del Valle del Najerilla II. Gobierno de la Rioja : Consejena de Educacion Cultura y Deportes, Logrono. (1991) $400 \mathrm{pp}$.

[15] - P. Ondina, II Gasteropodos terrestres de A Coruna y Pontevedra II. Tesis Doctoral. Universitat De Santiago. (1988) $386 \mathrm{p}$.

[16] - A. Martinez - Orti, ॥ Moluscos terrestres testaceos de la comunidad Valenciana II. Tesis Doctoral, Universitat de Valencia, (1999) 743 p.

[17] - L. Bigot et P. Aguesse, II Considération sur les adaptations de la faune des Invertébrés aux conditions particulières de fonctionnement des écosystèmes d'un Delta méditerranéen (la Camargue ou delta du Rhône) II. Bul. Muséum d'Histoire Naturelle, Marseille, 44 (1984) 7-17.

[18] - S. Bechlaghem, II Contribution à l'étude bioécologique de la faune retrouvée dans deux stations (Sebdou, El-Aricha) (Wilaya de Tlemcen) II. Thèse Ingéniorat en Ecologie et Environnement. Département de Biologie. Faculté des Sciences. Université Aboubekr Belkaid-Tlemcen- (2005) $100 \mathrm{p}$.

[19]- A. Damerdii, " Contribution à l'étude biosystématique des Mollusques Gastéropodes Pulmonés terrestres de la région de Tlemcen II. Thèse Magister, Institut de Biologie, Université de Tlemcen (1990) 205 p.

[20] - R. Daj0z, Précis d'écologie. Ed. Bordas, Paris, (1985) 505 p.

[21] - L. Germain, Mollusques terrestres et fluviatiles. Kraus, Nendeln, Liechtenstein, $21\left(1969_{\mathrm{a}}\right) 477 \mathrm{pp}$.

[22] - L. Germain, Mollusques terrestres et fluviatiles. Kraus, Nendeln, Liechtenstein, $22(1969$ b) $240 \mathrm{pp}$.

[23] - A. Damerdii, ॥ Bioécologie de la malacofaune retrouvée dans 2 stations (Hafir et Zarifelt) des Monts de Tlemcen II. Communication Orale. Colloque méditerranéen 
sur la gestion durable des espaces montagnards. Université Aboubekr Belkaid, Tlemcen, 10 et 11 octobre 2004. 14 p. (2004 .).

[24] - A. Damerdji, I Répartition des Mollusques Gastéropodes terrestres du littoral vers la steppe dans la région de Tlemcen II. Poster. Colloque méditerranéen sur la gestion durable des espaces montagnards. Université Aboubekr Belkaid, Tlemcen, 10 ef 11 0ctobre 2004. 14 p. $\left(2004_{\mathrm{b}}\right)$.

[25] - L. Bigot, II Essai d'Ecologie quantitative sur les Invertébrés de la Sansouire camarguaise II. Imprimerie M. DECLUME, Lons-Le-Saunier, (1965) 100 p.

[26] - A. Damerdji, II Impact des facteurs abiotiques et biotiques sur la taille des coquilles de Sphincterochila candidissima (Mollusca -Sphincterochilidae) dans la région de Tlemcen (Algérie) II. Revue I.N.R.A.A., 9 (2001) 101-109.

[27] - P. Aguesse et L. Bigot, II Complément à l'inventaire de la faune camarguaise : les Mollusques terrestres et des eaux douces et saumâtres 11. ( $5^{\text {ème }}$ note). Extrait de Terre et Vie, 1 (1962) 82-90.

[28] - J. Altes, I Sur le polymorphisme de la coquille de L. candidissima, modalités et déterminisme II. Bulletin Muséum d'Histoire Naturelle, Marseille, 16 (1956) 53-67.

[29] - H. Engel, "Okologisch-faunistiche Studien im Rhône-Delta, unter besonderer Berï chsichtigung der Mollusken", Bon. Zool. Beitr, VIII (1) (1957) 5-55.

[30] - L. Bigot, II Un microclimat important de Camargue : les coquilles vides de Mollusques II. Terre et Vie, (1957) 253-258.

[31] - C. F. Sacchi, " Ecologie comparée des Gastéropodes Pulmonés des dunes méditerranéennes et atlantiques II. Natura, Milan, 62 (1971) 277-358. 